\title{
Dielectric and relaxation processes in PEG based polymeric networks
}

\author{
Harishankar Kaushik ${ }^{1}$, Mamta Sharma ${ }^{1}$, Anuradha Sharma ${ }^{2}$, A K Tiwari ${ }^{3}$, \\ ${ }^{1}$ Department of Humanities \& Applied Sciences, YMCA University of Science \& Technology, Faridabad, \\ Haryana, INDIA \\ ${ }^{2}$ Department of Physics and Material Science \& Engg. PEC University of Technology, Chandigarh, India \\ ${ }^{3}$ DCRS, INMAS, Delhi-110054, India
}

\begin{abstract}
Acrylics are sometimes referred to as "plastic glass" which have not only captured the novelty trade for costume jewelry, pocketbooks, hairbrushes, umbrella handles, trays and ornaments, but also have found more practical purposes in many industrial and commercial products for everyday use.
\end{abstract}

\section{Introduction}

The industrial applications of solutions or emulsion of acrylic ester polymer as coating or impregnants are many and varied. The inherent stability, durabilty and pigment binding characteristics of acrylic polymers have been important factors in selection of such systems in various coating applications such as in leather and textile finishing. Acrylic ester emulsion polymers are used in the paper industry as pigment binders, saturants, fibers and wet-end additions for high strength paper [1-7]. Biomedical applications of acrylate based hydrogels include soft contact lenses, artificial corneas, soft tissue substitutes and burn dressings. Furthermore, the application of hydrogels to a variety of substrates leads to the production of thrombo-resistant coatings, catheters or blood detoxicants. Hydrogels are impregnated with biologically active agents, such as antibiotics, enzymes, contraceptives, drug antogonists, anticancer drugs etc. and may serve as systems for the controlled release of the agents absorbed to a specific size of the body[8-12]. The acrylate and methacrylate esters provide a series of monomers whose polymers vary from soft, rubberlike materials to hard plastics. Polymethyl acrylate is a tough, rubbery and moderately hard polymer and having little or no tack at room temperature. Polyethyl acrylate is considerably softer, more extensible, more rubberlike and not quite as tough as polymethyl acrylate. The polymer of n-butyl acrylate is still softer and rather tacky at room temperature. These trends to tackier, softer polymer continue to n-dodecyl (lauryl) ester [13-20].

Dielectric relaxation properties of PMA hydrogel were examined by Gomez et. al. [21-22]. They explained that PMA presents two relaxation zones, labelled $\gamma$ and $\alpha$, when it is completely dry. The temperature of the maximum of the $\gamma$ relaxation, as well as its apparent activation energy, are somewhat higher than in poly(ethyl metharylate), PEMA, a fact that could be explained by higher intermolecular interactions in the series of polyacrylates than in the series of polymethacrylates. The absorption of even slight traces of water causes a new relaxation to appear. The intensity of which increases with the content of water, while at the same time the intensity of the $\gamma$ relaxation decreases. This fact suggests the formation of an association of the water molecules with the polar groups of the side chains in the polymer, in such a way that the internal motions in the group resulting from this association are further hindered, producing a different relaxation.

The characterization of the $\alpha$ relaxation is difficult because of the high d.c. conductivity component of the permittivity. Its temperature suggests the presence of hydrogen bonds, which render the main chains rigid. Depolarization thermocurrent and dielectric relaxation spectroscopy in PMA/water hydrogels were studied by Kyritsis et. al. [23-26]. PMA Hydrogels with water contents varying in a wide interval were studied by differential scanning calorimetry (DSC) and thermally stimulated depolarization currents (TSDC). The TSDC measurements reveal the influence of water on the secondary relaxations when the water content is higher than $0.3 \%$. The dependence of the glass transition temperature and the temperature of the main dielectric relaxation had been determined as a function of the water content. Detailed investigations on the dielectric relaxation mechanisms in PMA, by means of the TSDC method in the temperature range 77-300 K were reported. There was particular interest in the dependence of the dielectric relaxation mechanisms on the water content, $\mathrm{h}, \mathrm{h}=0-0.5 \mathrm{w} / \mathrm{w}$, in an attempt to contribute to a better understanding of the physical structure of water in the PMA hydrogels.Permeability in the amorphous state of polyacylates increase as the side chain length becomes longer but shows mixed trends in the crystalline state. Permeability decreases in the amorphous state when the backbone stiffens. The effect of the main chain versus side chain crystallinity on the permeation is more. The influence of a compositional change in the amorphous phase upon melting on the gas permeability of sidechain crystalline polymers is also considerable [27-30]. 


\section{Experimental Details}

Commercial Methyl acrylate always contains impurities, such as acrylic acid and ethylene glycol diacrylate (EGDA). Purification has to be done to remove diacrylate and acrylic acid. The presence of diacrylate leads to crosslinking and in the presence of large amounts of acrylic acid, no polymerization is observed. Therefore, polymerizations of unpurified or poorly purified monomer may be either incomplete and slow or lead to insoluble solids. MA (Acros, $97 \%$ ) was dissolved in water (25\% by volume) and the solution was extracted 10 times with hexane to remove diacrylates. The aqueous solution was then salted $(200 \mathrm{~g} \mathrm{of} \mathrm{NaCl} / \mathrm{L})$ and the monomer was separated from the aqueous phase by ether extraction (4 times) to remove acrylic acid. Finally, MgSO4 (3 wt \%) drying agent was used to remove traces of water before evaporation of the ether phase in a rotary evaporator. The purified monomer was distilled under vacuum immediately prior to use for polymerizations.Methanol, carbontetrachloride (Merck), hexane (Merck), diethyl ether (Riedel de-Haen), benzyl chloride (Aldrich 99\%) were all reagent grades and used without further purification.

\section{INSTRUMENTATION}

\section{PolymerizationTubes}

The tubes were $1-3 \mathrm{~cm}$. in diameter, $10 \mathrm{~cm}$. in length Pyrex tubes. The open ends of the pyrex tubes were attached to another tube of smaller diameter, which facilitates connection to the vacuum line using fittings.

\section{High Vacuum System}

In order to evacuate the vacuum tubes containing the monomer, high vacuum system at $10^{-4}$ to $10^{-5} \mathrm{mmHg}$ pressures for about 5-6 hours was applied.

\section{a) Duo-Seal Vacuum Pump}

It is a product of "Sargent-Welch Scientific Co." Model 1399 and capable of pressure reduction down to 1.5 x $10^{-2} \mathrm{mmHg}$

\section{b) Mercury Diffusion Pump}

It is a water-cooled one-stage diffusion pump with an approximate capacity of $200 \mathrm{ml}$ of mercury. Mercury was heated by a $143 \mathrm{~W}$ metallic bond resistive heater operating at $130 \mathrm{~V}$, which is a product of "Pliz Co." type 62.

\section{c) Main Manifold}

A Pyrex glass tube of length $110 \mathrm{~cm}$., diameter $4.53 \mathrm{~cm}$. was employed. It was connected to the first trap by a high vacuum cockstop and to the sample holder tubes to high vacuum cockstops with standard joints.

\section{d) Liquid Nitrogen Traps}

Two Pyrex traps were used to protect the pumps from the chemicals evaporating at low pressures and placed before the connection of each pump.

Polymerization of PMA was done under vacuum in three different modes:

(a) Bulk Polymerization at open air

(b) Solution Polymerization

(c) Atomic Transfer Radical Polymerization

\section{Tensile Tests}

Tensile tests were performed by a LLOYD LR 5K Computerised testing machine for all specimen groups. Tests were performed according to ASTM D638M-91a, (Standard Test Method for Tensile Properties of Plastics). The speed was $20 \mathrm{~mm} / \mathrm{min}$. The shape and the dimensions of the specimens are illustrated in Figure 2.1.

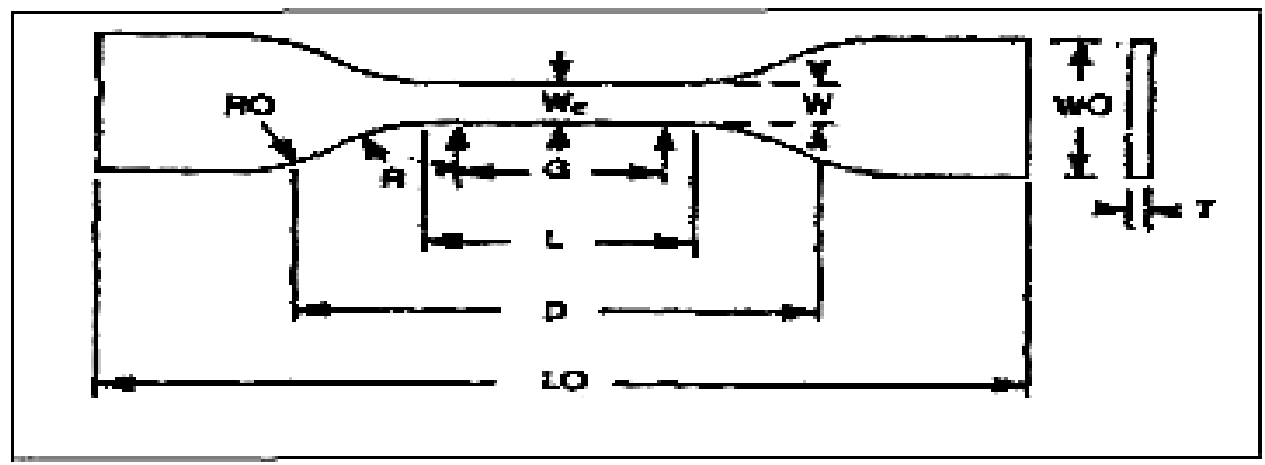

Figure 1 tensile Test specimens 
$\mathrm{W}=$ Width of narrow section

$\mathrm{L}=$ Length of narrow section

W0 $=$ Width overall $(3.00 \mathrm{~mm})$

LO = Length overall

$\mathrm{G}=$ Gauge length $(3.10 \mathrm{~cm})$

$\mathrm{D}=$ Distance between grips

$\mathrm{T}=$ Thickness $(2.70 \mathrm{~mm})$

$\mathrm{W}_{\mathrm{c}}=$ Width at the center

\section{Film Preparation}

After the polymer powder was converted, it dissolved into solvents such as Chloroform, DMF. Of these solvents, DMF dissolves the largest amount of PMA powder. Therefore, DMF was chosen to make a solution of the processed PMA powder which was prepared by the above procedures. The polymer-solvent solution was used to cast films. The solvent which was used for preparing polymer solutions to be cast into thin films.

\section{Thickness Meassurement}

The thickness $(\mathrm{mm})$ of the films prepared in this study were measured by the digimatic caliper, Model CD-8"P made by Mititoyo Corporation. The smallest readable digit is $0.01 \mathrm{~mm}$. First, the caliper is put on the films and left idle for more than one second. This is because the caliper has a start-up time of approximately one second. Also, before measuring the thickness of the film, the caliper needs to be adjusted to zero it. This is done by pressing the ON/ZERO switch when the two outside measuring faces touch each other. The film is then placed between the outside measuring faces. The thumb roller is slowly moved until the two outside measuring faces clipped the film. The caliper should not be disturbed so as to get accurate readings.

\section{Electrode Coating}

Prepared thin film with $25 \mu \mathrm{m}$ thickness and used in form of square sheets $\left(1.0 \mathrm{~cm}^{2}\right)$. The samples were vacuum evaporated with $100 \mathrm{~nm}$ thickness of electrodes and $1 \mathrm{~cm}$ diameter to opposite sides and had been previously polished using polishing rouge and dry methanol. Evaporated electrodes were used to prevent an air gap trapping between the electrode and the sample and to increase ohmic contact, TSDC measurements were carried out with an apparatus developed in our laboratory from the concept outlined by Bucci and Fieschi and described elsewhere.

\section{Result And Discussion}

The FT-IR spectra obtained for doped polymethyl acryl ate is shown in graph3 (a) .In the IR spectrum of monomer, a characteristic carbonyl peak $(\mathrm{C}=\mathrm{O}$ stretching $)$ is at $1712 \mathrm{~cm}^{-1}$. The peaks for the $\mathrm{C}=\mathrm{C}$ was observed at $1638 \mathrm{~cm}^{-1}$ and at $935 \mathrm{~cm}^{-1}$. The peaks in the range of $2900-3000 \mathrm{~cm}^{-1}$ correspond to the aliphatic $\mathrm{C}-\mathrm{H}$ stretching. The -CH2- peak is at $1410 \mathrm{~cm}^{-1}$. The peak at $3415 \mathrm{~cm}^{-1}$ corresponds to $\mathrm{O}-\mathrm{H}$ stretching. Furthermore, O-H bending was observed at $1298 \mathrm{~cm}^{-1}$. The ester peak (C-O stretching) of monomer can be seen at $1200 \mathrm{~cm}^{-1}$.

In the spectrum of PMA, $\mathrm{C}=\mathrm{O}$ bond stretching appeared at $1719 \mathrm{~cm}^{-1}$. Also an aliphatic $\mathrm{C}-\mathrm{H}$ stretching was observed at $2946 \mathrm{~cm}^{-1}$ and an aliphatic $\mathrm{C}-\mathrm{H}$ bending $\left(-\mathrm{CH}_{2^{-}}\right)$at $1447 \mathrm{~cm}^{-1}$. The broad peak at $3500 \mathrm{~cm}^{-1}$ corresponds to $\mathrm{O}-\mathrm{H}$ bond stretching and also $\mathrm{O}-\mathrm{H}$ bending can be seen at $1394 \mathrm{~cm}^{-1}$. The ester peak of polymer was observed $1162 \mathrm{~cm}^{-1}$. Peaks belong to double bond were completely disappeared.

The DSC measurements were carried out at N2 atmosphere by heating polymer samples in the temperature range of -150 to $300{ }^{\circ} \mathrm{C}$. No melting peak of polymer was observed due to the amorphous structure. The values are given in plot. The thermal stability of PMA samples were also characterized by means of TGA from $30^{\circ} \mathrm{C}$ to $600^{\circ} \mathrm{C}$ in $\mathrm{N}_{2}$ atmosphere as illustrated in Figure 3.3. From the TGA thermograms, two measured weight losses were seen for bulk PMA's in which gamma and BPO were used as initiator. The thermograms are typical for acrylate polymers that thermal degradation is in the form of depolymerization. The small peak at $402{ }^{\circ} \mathrm{C}$ for polymerization by radical initiator and a similar broad peak for polymer obtained by $\gamma$-radiation show the degradation of oligomers and/or residual monomer that may exist in crosslinked polymer. This peak is not observed for polymer obtained by solution polymerization. The main decomposition peak is much sharper and appears at lower temperature for the polymer obtained in solution. The broad peaks and higher temperature in first two figures show higher molecular weights and crosslinking or larger dispersive index of polymers. The TSDC thermo gram obtained for poling temperature with different values of polarizing field . These characteristics are showing two peaks, one at low temperature ( $\beta$ peak), which is associated with dipolar relaxation, and other at higher temperature ( $\alpha$ peak) that appears due to space charge relaxation. The analysis of the shape of TSDC peak makes it possible to obtain the activation energy, relaxation time and charge released. When polarizing field was increased, the magnitudes of both peaks increased. 
The present study on polarizing field and temperature dependence of electrical conduction in undoped and doped films of PMA has been carried out to understand the role of dopant when added in varying concentrations to a polymer matrix. In the present study, tri subsituted ethylene glycol is a dopant, doped in different weight $\%(5 \%, 10 \%, 20 \%, 30 \%)$ in PS. But results on only two concentrations $(5 \%, 30 \%)$ are discussed in detail because they show a remarkable effect on conduction It is observed that d.c. conductivity of undoped PMA has been enhanced by dopant even if it is in lower concentration. But as the concentration increases, conductivity increases and it reaches to maximum value for higher concentration of dopant As the dopant concentration is increased, the dopant molecules start bridging the gap separating the two localized states and lowering the potential barrier between them, thereby facilitating the transfer of charge carrier between two localized states.

It is well known that electrical polarization in polymers is caused by the dipole alignment, space charge injected into the polymer by surface breakdown, charge injection from the electrodes, rotation or motion of the main or segmental polymer chain, orientation of permanent dipole, etc. The TSDC of shows that the lowtemperature peak is possibly due to the motion of dipolar unit corresponding to $b$ relaxation Work reported earlier by (Herman and Rembaum 1967; Davies and Lock 1973) \{54-58\}suggests the formation of $1: 1$ charge transfer (CT) complex between donor MA and acceptor Dopant The formation of CT complex effectively reduces the trapping effect. A CT complex in the present context means an interaction between two species, the strength of which lies between a full chemical bond and a small orbital overlap. The formation of CT complex between MA and dopant at an optimum concentration is evident from the sudden increase in the value of conductivity at higher concentration. The conductivity of polymer also depends on temperature. As the temperature increases polymer becomes soft and mobility of the main chain segments as well as the rotation of side groups become easier. Thus, at higher temperature more and more dipoles are oriented resulting in the higher equivalent surface charge density i.e. as temperature increases conductivity increases as per Arrhenius equation $\sigma=\sigma_{0} \exp (-\mathrm{Ea} / \mathrm{KT})$

where $\sigma_{0}$ is the pre-exponential factor, Ea the activation energy of conduction and $\mathrm{K}$ the Boltzmann's constant. The activation energy for these low and high temperature regions are also different

According to the classical theory of Perlman and others, the total charge of the electret is the superposition of homocharge and heterocharge. Homocharge is produced by the discharge in the air gap between the poling electrode and the surface of the sample and also by the injection of charge carriers from the poling electrode into the sample. Thus, homocharge is the space charge consisting of ions and electrons. The enhancement of depolarization current with Ep at each Tp may also be considered to indicate that the build-up of polarization is due to charge penetration though the non-crystallized surface into the near surface region of the sample and its subsequent trapping in various available traps. Conduction in lower temperature region takes place by thermal activation of carrier from one disordered state to another. At higher temperature, the traps are immobilized because of segmental chain motion or entire chain motion and agrees well with that reported by other workers (Sinha and Srivastava 1979; Tiwari et al 1987). This could only be explained if we assume that dopant molecules when present in sufficient quantity form a link between trapping centres because of increased orbital overlaps. These experimental findings lead one to understand that steric effects in polymeric CT complex play an important role. Rembaum et al(1968) suggest that in the polymer system, the acceptor cannot get close to the donor. It seems likely that in many polymeric CT systems, the bulky nature of the groups and their close proximity to the polymer backbone will prevent the maximum interaction and stocking of acceptor layers is characteristic of the highly conducting naphthalene. Steric effects may place a serious limit upon the extent to which conductivity can be enhanced by CT complex formation as evident from the present observations.

\section{Conclusion}

Polmethyl acrylate (pma) is an important conducting polymer that has been extensively studied because of its various application in industries.the influence of the polymerization temperature on electrical conductivity of pma has been investigated. The $\%$ change of this thin film as per the morphology of polymer changes from granular (reaction in a liquid medium) to macroporous (polymerization in the frozen state). Commercially available polymer used in the present work is amorphous and the dopant used viz. tri substituted ethylene glycol, has an electron donating hydrocarbon group which may act as a trapping centre for charge carriers.

\section{References}

[1] Coca, C. B. Jasieczek, K. L. Beers, K. Matyjaszewski, Journal of Polymer Science, Part A, 36, 1417-1424 (1998).

[2] Calvin E. Schildknecht, Vinyl and Related Polymers, John Wiley \& Sons Inc., New York, 1959.

[3] E. H. Riddle, Monomeric Acrylic Esters, Reinhold Publishing Corp., New York, 1954

[4] A.N., Akkuş, M.Sc. Thesis, METU, September 1988.

[5] Caliceti P., Veronese F., Schiavon O., Lora S., Carenza M., Farmaco, 47 (3), 275-286 (1992). 
[6] Inukai M., Jin Y., Yomota C., Yonese M., Chemical and Pharmaceutical Bulletin, 48(6), 850-854 (2000).

[7] Mengoli G., Martina A., Furlanetto F., Musiani M., Chimica L Industria, 62(1), 16-22 (1980).

[8] Zubarev E., Talroze R. V., Plate N. A., Vysokomolekulyarnye Soedineniya Seriya A \& Seriya B, 39(6), 1031-1037 (1997).

[9] M. Pradas, J.L.G. Ribelles, A.S. Aroca, G.G. Ferrer, Polymer, 42, 4667-4674 (2001).

[10] J.L.G. Ribelles, J.M.M. Duenas, M.M. Pradas, Polymer, 29, 1124-1127

[11] Kyritsis, P. Pissis, J.L.G. Ribelles, M.M. Pradas, Journal of Polymer Science, Part B, Polymer, Phys., 32, 1001-1008 (1994).

[12] Z. Mogri, D.R.Paul, Polymer, 42, 7765-7780 (2001).

[13] T. Itoh, Y. Ohkawa, K. Ishihara, Polymer Journal, 15, 827-834 (1983).

[14] M.M. Pradas, J.L.G. Ribelles, A.S. Aroca, G.G. Ferrer, J.S. Anton, P.Pissis, Colloid Polymer Science, 279, 323-330 (2001).

[15] D. Mangaraj, S.K.Patra, Die Makromolekulare Chemie, 107, 230-240 (1967).

[16] Stanley R. Sandler, Wolf Karo, Polymer Synthesis, Vol. 1, Academic Press,New york and London, Chapter 10,1974.

[17] Robert W. Lenz, Organic Chemistry of Synthetic High Polymers, Interscience Publishers, Chapter 11, 1967.

[18] Kelly A. Davis, Krzysztof Matyjaszewski, Statistical, Gradient, Block and Graft Copolymers by Controlled/Living Radical Polymerizations, Springer-Verlag Berlin, 2002.

[19] Scott G. Gaynor, Ph. D. Thesis, Carnegie Mellon University, April 1997.

[20] Fred W. Billmeyer, Textbook of Polymer Science, Interscience Publishers, New York, Chapter 9, 1962.

[21] R. J. Young and P.A. Lovell, Introduction to Polymers, Chapman \& Hall, London, 2nd edition, Chapter 2, 1991.

[22] Gloria Gallego Ferrer, Dr. Eng. Sci. Thesis, Valencia, 2001.

[23] J. Rault, A. Lucas, R. Neffati and M.M. Pradas, Macromolecules, 30, (1997).

[24] J.L.G. Ribelles, M.M. Pradas, G.G. Ferrer, N.P. Torres, V.P. Gimenez, P.Pissis, A. Kyritsis, Journal of Polymer Science, Part B, Polymer Phys., 37, 1587-1599 (1999).

[25] C.F. Degiorgi, R.A. Pizarro, E.E. Smolko, S. Lora, M. Carenza, RadiationPhysics and Chemistry, 1-5 (2001).

[26] Mano JF, Gomez Ribelles JL, Gomez Tejedor JA. Polymer 2004;45(3): 1007-17. 